PROCEEDINGS OF THE

AMERICAN MATHEMATICAL SOCIETY

Volume 134, Number 10, October 2006, Pages 3057-3059

S 0002-9939(06)08460-7

Article electronically published on May 5, 2006

\title{
HYPERBOLIC SURFACES WITH PRESCRIBED INFINITE SYMMETRY GROUPS
}

\author{
DANIEL ALLCOCK \\ (Communicated by Alexander N. Dranishnikov)
}

\begin{abstract}
For any countable group $G$ whatsoever, there is a complete hyperbolic surface whose isometry group is $G$.
\end{abstract}

Greenberg proved in 1972 [2] that for any finite group $G$ there is a closed hyperbolic 2-manifold having isometry group isomorphic to $G$. Kojima proved the corresponding result for hyperbolic 3-manifolds 3. Following work of Long and Reid 4, Belolipetsky and Lubotzky extended this to hyperbolic manifolds of arbitrary dimension 1. Our purpose here is to give a generalization in a different direction, which also provides an elementary proof of Greenberg's original theorem.

Theorem. For any countable group $G$, there is a complete hyperbolic surface $X$ having isometry group isomorphic to $G$. When $G$ is finite, $X$ may be taken to be closed.

We build $X$ by gluing together pairs of pants. A pair of pants is a surface homeomorphic to the 2-sphere minus three open disks whose closures are disjoint, equipped with a hyperbolic metric under which the boundary curves are geodesic. (All boundary curves in this note are assumed geodesic.) Up to isometry, there is a unique pair of pants whose boundary lengths are any three given positive numbers. The standard way to build pairs of pants is by doubling right-angled hexagons; see [5. This construction makes the following lemma obvious.

Lemma. There exists $\varepsilon>0$ such that if $P$ is a pair of pants with all its boundary curves of length $<\varepsilon$, then

(1) the boundary curves are the only closed geodesics on $P$ of length $<\varepsilon$, and

(2) any geodesic interval in $P$ of length $<\varepsilon$ with its endpoints in $\partial P$ lies entirely in $\partial P$.

We call a closed geodesic on a hyperbolic surface short if it has length $<\varepsilon$, and very short if it has length $<\varepsilon / 2$. The point of the lemma is that on a hyperbolic surface obtained by gluing together pairs of pants with all short boundary curves, the short curves are exactly the curves along which the pairs of pants were glued.

Received by the editors May 4, 2005.

2000 Mathematics Subject Classification. Primary 51M09; Secondary 20 F99.

This work was partially supported by NSF grant DMS-024512.

(C)2006 American Mathematical Society Reverts to public domain 28 years from publication 3057 
(1) deals with short closed geodesics that lie in a single pair of pants and (2) rules out the existence of any others.

Proof of the Theorem. Let $I$ be a set indexing a sequence of group elements $a_{i}$ that generate $G$; by enlarging $I$ we may suppose $|I| \geq 2$. The Cayley graph $C$ of $G$ has automorphism group equal to $G$, and we will use $C$ as a sort of framework on which to build $X$. Explicitly, $C$ has vertex set $G$, and for each $g \in G$ and each $i \in I$ there is a directed edge from $g$ to $g a_{i}$ labeled ' $i$ '. An automorphism of $C$ is an automorphism of the underlying graph which preserves the directions and labels of edges. $G$ acts on $C$ by left-multiplication, and it is easy to see that $G$ is all of Aut $C$. (An automorphism $g$ of $C$ permutes the vertices in a manner commuting with $G$ 's right-multiplication action on itself; since this action is transitive, $g$ is completely determined by what it does to any one vertex.)

We choose positive numbers $\alpha_{i}, \beta_{i}<\varepsilon / 2$ for $i \in I$, all distinct. We build for each $i$ a compact hyperbolic surface $E_{i}$ with two boundary curves, of lengths $\alpha_{i}$ and $\beta_{i}$. We may suppose that these are the only very short curves on $E_{i}$, say by building $E_{i}$ from two pairs of pants, with boundary curves of lengths $\alpha_{i}, \varepsilon / 2$ and $\varepsilon / 2$ (resp. $\beta_{i}, \varepsilon / 2$ and $\varepsilon / 2$ ), glued together along their boundary curves of length $\varepsilon / 2$. We call $E_{i}$ the $i$ th 'edge surface'.

Next we build a complete hyperbolic surface $V$ with $2 \cdot|I|$ boundary curves, of lengths $\alpha_{i}$ and $\beta_{i}$, by gluing a sequence of pairs of pants together along boundary curves which are short but not very short. The shortness of the boundary curves of the pairs of pants assures us that the only very short geodesics on $V$ are its boundary curves. We may suppose that each of the pairs of pants has boundary components of three distinct lengths; this lets us arrange for $V$ to have trivial isometry group, as follows. $\operatorname{Isom}(V)$ must permute the short geodesics of $V$, hence permute the pairs of pants, and hence carry to itself the unique one of these pairs of pants with a boundary curve of length $\alpha_{0}, 0$ being some fixed element of $I$. A pair of pants with boundary curves of three distinct lengths has isometry group $\mathbb{Z} / 2$, and by rotating the gluing of an adjacent pair of pants, we can make sure that the nontrivial isometry does not extend to an isometry of $V$. We call $V$ the 'vertex surface'.

Now we build $X$. We take one copy of the vertex surface for each vertex of $C$ and attach one copy of the edge surface $E_{i}$ for each edge of $C$ labeled $i$. We attach the length $\alpha_{i}$ (resp. $\beta_{i}$ ) boundary curve of each copy of $E_{i}$ to the length $\alpha_{i}$ (resp. $\beta_{i}$ ) boundary curve of the copy of $V$ from which the edge originates (resp. to which it points). This may be done in a manner compatible with the action of $G$ on $C$, so that $G \subseteq$ Isom $X$.

To see that $G$ is all of Isom $X$, suppose $g: X \rightarrow X$ is an isometry. Then it permutes the very short geodesics of $X$ and hence the components of their complement, which are the (interiors of the) edge and vertex surfaces we used to build $X$. It must carry components with two boundary curves to other such components, so it carries edge (resp. vertex) surfaces to edge (resp. vertex) surfaces. (This is where we use the assumption $|I| \geq 2$.) Since the $\alpha_{i}$ and $\beta_{i}$ are all distinct, $g$ carries edges labeled $i$ to other such edges, and preserves their directions. Therefore $g$ acts on $C$; by composing it with an element of $G$ we may suppose that $g$ fixes a vertex of $C$. Since we chose $V$ to have no isometries, $g$ must be the identity.

It is clear that $X$ is complete; it may be taken to be compact when $G$ is finite by taking $I$ finite and $V$ compact. 


\section{ADDED IN PROOF}

The author has learned that J. Winkelmann has proven essentially the same result, by a totally different argument. (J. Winkelmann, Realizing countable groups as automorphism groups of Riemann surfaces, Documenta Math. 7 (2002) 413-417.)

\section{REFERENCES}

[1] M. Belolipetsky and A. Lubotzky, Finite Groups and Hyperbolic Manifolds, Invent. Math. 162 (2005) 459-472. MR2198218

[2] L. Greenberg, Maximal groups and signatures, Discontinuous groups and Riemann surfaces (Proc. Conf., Univ. Maryland, College Park, Md., 1973), Ann. of Math. Studies, No. 79, Princeton University Press, 1974, pp. 207-226. MR0379835 (52:740)

[3] S. Kojima, Isometry transformations of hyperbolic 3-manifolds, Topology and its Applications 29 (1988) 297-307. MR0953960 (90c:57033)

[4] D. D. Long and A. W. Reid, On asymmetric hyperbolic manifolds, Math. Proc. Camb. Phil. Soc. 138:2 (2005) 301-306. MR2132171

[5] W. Thurston, Three-dimensional geometry and topology, ed. Silvio Levy, Princeton Mathematical Series, 35, Princeton University Press, 1997. MR1435975 (97m:57016)

Department of Mathematics, University of Texas at Austin, Austin, Texas 78712

E-mail address: allcock@math.utexas.edu

$U R L$ : http://www. math.utexas.edu/ allcock 